\title{
Unusual Facilitated Olefin Transport through Polymethacrylate/Silver Salt Complexes
}

\author{
Jong Hak Kim, Seung Hwan Joo, Chang Kon Kim, and Yong Soo Kang* \\ Center for Facilitated Transport Membranes, Korea Institute of Science and Technology, \\ P. O. Box 131, Cheongryang, Seoul 130-650, Korea \\ Jongok Won \\ Department of Applied Chemistry, Sejong University, Seoul 143-747, Korea
}

Received Aug. 12, 2003; Revised Sept. 5, 2003

\begin{abstract}
Silver salt complex membranes with glassy poly(methyl methacrylate) (PMMA) unexpectedly showed higher propylene permeance than those with rubbery poly(butyl methacrylate) (PBMA) where as neat PMMA is much less permeable to propylene than that of neat PBMA. Such unusual facilitated olefin transport has been systematically investigated by changing the side chain length of polymethacrylates (PMAs) from methyl, ethyl to butyl. The $a b$ initio calculation showed almost the same electron densities of the carbonyl oxygens in the three PMAs, expecting very similar intensity of the interaction between carbonyl oxygen and silver ion. However, the interaction intensity decreases with the length of the alkyl side chain: PMMA > PEMA > PBMA according to wide angle X-ray scattering and FT-Raman spectroscopy. The difference in the interaction intensity may arise from the difference in the hydrophilicity of the three PMAs, as confirmed by the contact angle of water, which determines the concentrations of the ionic constituents of silver salts: free ion, contact ion pair and higher order ionic aggregate. However, propylene solubilities and facilitated propylene transport vary with the side chain length significantly even at the same concentration of the free ion, the most active olefin carrier, suggesting possible difference in the prohibition of the molecular access of propylene to silver ion by the side chains: the steric hindrance. Therefore, it may be concluded that both the hydrophilicity and the steric hindrance associated with the side chain length in the three PMAs are of pivotal importance in determining facilitated olefin transport through polymer/silver salt complex membranes.
\end{abstract}

Keywords: silver ion, facilitated transport, olefin, side chain, polymethacrylate.

\section{Introduction}

Polymer/metal salt complexes have attracted considerable attention because of their versatile applications to secondary batteries, fuel cells, electrochromic displays and etc. ${ }^{1-3}$ Low lattice-energy salts are dissolved in a polymeric ligand containing oxygen, nitrogen or sulfur to form polymer/metal salt complexes by coordinative interaction between the ligand and metal ion. Polymer/silver salt complexes have also been much interested recently because they can be used successfully as facilitated transport membranes in solidstate for olefin/paraffin separation. ${ }^{4-19}$ They have offered a promise for highly efficient membranes to separate olefin/ paraffin mixtures. For example, $99 \%$ pure propylene con-

*e-mail : yskang@ kist.re.kr

1598-5032/10/375-07 @ 2003 Polymer Society of Korea centration can be obtained by a single pass of silver polymer electrolyte membranes when a feed stream concentration is $50 \%$ propylene. $^{4}$

The silver polymer electrolyte membranes consisting of silver ions dissolved in poly(2-ethyl-2-oxazoline) (POZ) or poly $(N$-vinyl pyrrolidone) (PVP) showed very high separation performance for olefin/paraffin mixtures. ${ }^{4-9}$ The propylene permeance increases up to almost $45 \mathrm{GPU}$ at $140 \mathrm{kPa}$ feed pressure with increasing silver ion content, while the propane permeance is reduced to as low as $0.003 \mathrm{GPU}{ }^{4,8}$ Thus, the ideal separation factor of propylene over propane is approximately 15,000 . The maximum actual selectivity for gaseous mixtures, defined by the ratio of mole fractions of the gas components in permeate and feed streams, is higher than 50. ${ }^{9}$ This exceptional performance enhancement is achieved primarily due to 1) extremely high loading of silver salts in the polymer matrix, which is possible by the 
interaction between silver ions and carbonyl oxygens of polymer matrices, ${ }^{4}$ and 2) a fast reversible reaction of silver ions with olefin. ${ }^{10}$

In a series of previous studies, we obtained results that shed light on the mechanism of facilitated olefin transport through silver polymer electrolyte membranes. ${ }^{7-9,19}$ First, the coordinative interaction between silver ion and polymeric ligand induces the dissolution of silver salts in a polar polymer solvent. Its intensity and silver salt concentration in the polymer matrix determine the concentrations of ionic constituents such as free ion, ion pair and higher-order ionic aggregates. It is spectroscopically observed that all three silver ionic constituents are converted into free ions under an olefin environment, suggesting that the most effective olefin carrier is the free silver ion. ${ }^{7}$ On these experimental findings, a new mechanism for the complexation reaction between propylene and silver salt in silver polymer electrolytes was proposed. $^{7}$

Second, in facilitated olefin transport through silver polymer electrolytes, a threshold concentration was observed at the silver mole fraction of approximately 0.25 for $\mathrm{AgBF}_{4}$ or $\mathrm{AgCF}_{3} \mathrm{SO}_{3}$ dissolved in POZ or PVP, below which facilitated transport was not observed. ${ }^{8,9}$ However, the propylene permeance increased nearly linearly with the silver concentration at silver concentrations above the threshold concentration. The existence of the threshold concentration may be attributable to the coordination behavior of silver ion with polymeric ligand as well as olefin. For $\mathrm{AgBF}_{4}$ or $\mathrm{AgCF}_{3} \mathrm{SO}_{3}$ $/ \mathrm{POZ}$ or PVP systems, ${ }^{8}$ it was found that 1 ) the most favorable coordination number of silver ion under a propylene environment was 3 , and 2 ) the interaction between silver ion and amide carbonyl oxygen was marginally stronger than that between silver ion and olefin. It was thus proposed that a silver ion can act as an olefin carrier only when it has vacant coordination sites, i.e., when the number of amide carbonyl oxygens coordinated to the silver ion is less than 3 . It was further found that the threshold concentration for facilitated olefin transport was strongly dependent on the relative strength of the interactions of silver ion with the carbonyl oxygen and with olefin. ${ }^{9}$ The threshold concentration is high when the former interaction is slightly stronger than the latter, and is low when the latter is stronger than the former. This is the first report on the importance of the coordination behavior of metal ion in determining the activity of silver ion as olefin carrier, and the presence of the threshold concentration for facilitated transport membranes in solid state.

Third, the reaction mechanism between silver ion and olefin can be elucidated by calculating the theoretical structure of a silver-polymer complex in the gas phase and the electronic energies of its reaction step with olefin. ${ }^{19}$ The reaction between silver ion and olefin is proposed to occur by two steps; the first is to form a silver-olefin complex as an intermediate, and the second is the exchange of the com- plexed-olefin in the intermediate with a new olefin. The first complexation step is irreversible and slow, whereas the second exchange step is rapid and reversible, which appears to be the key step in determining facilitated olefin transport. Note that the second step is only observed when a concentration gradient is present, but not in a homogeneous system.

Despite the extensive research on facilitated olefin transport phenomena in the polymer/silver complex membranes, the relationship between the structure of macromolecular ligand and facilitated transport has been insufficiently explored. Accordingly, very little information has been obtained about the structural effect of polymer matrix, which is of pivotal importance in the development of enhanced performance in polymer/silver complex membranes. Among various polymer matrices containing oxygen, three different polymethacrylates (PMAs) such as poly(methyl methacrylate) (PMMA), poly (ethyl methacrylate) (PEMA) and poly(buthyl methacrylate) (PBMA) have been employed in this study because they have the same molecular structure containing methacrylate group except for the difference of side chain length of alkyl group. Especially the polymers having similar $M_{w}$ ranging from 337 to $350 \mathrm{k}$ have been chosen to avoid any possible steric effect by the different entanglement of polymer chains. Here we observed that silver salt complex membranes with glassy PMMA showed unexpectedly much higher propylene permeance than those with rubbery PBMA even though neat PMMA is much less permeable to propylene than neat PBMA. Thus we investigate the effect of macromolecular structure on facilitated olefin transport and the importance of side chain length of the ligand in determining the polymer/ silver salt complexation.

\section{Experimental}

PMMA $\left(M_{w}=350 \mathrm{k}\right)$, PEMA (340 k), PBMA (337 k), silver tetrafluoroborate $\left(\mathrm{AgBF}_{4}, 98 \%\right)$ and silver trifluoromethanesulfonate $\left(\mathrm{AgCF}_{3} \mathrm{SO}_{3}, 99+\%\right)$ were purchased from Aldrich Chemical Co. and were used without further purification. Polymer solution was prepared by dissolving $20 \mathrm{wt} \%$ polymer in tetrahydrofuran (THF, 99+\%, Aldrich). After complete dissolution, the predetermined amount of silver salt was added in the solution depending on the mole fraction of silver salt, i.e. $[\mathrm{Ag}] /([\mathrm{COO}]+[\mathrm{Ag}])$. The solution was then cast on teflon-attached glass plate and dried under $\mathrm{N}_{2}$ environment. The films were further dried in a vacuum oven for two days at room temperature.

Wide angle X-ray Scattering (WAXS) was utilized with $\mathrm{Cu}$ $K_{\alpha}$ radiation to determine the value of d-spacing in polymer/ silver complexes at a scanning speed of $5 \% \mathrm{~min}$. The contact angles for polymer surfaces were determined using a contact angle meter (Tantec, model CAM-Micro). The contact angle of deionized water on polymer surface was measured by the static drop method at room temperature. Each reported contact angle value is the average of at least six measurements. 
The IR spectra were obtained with a pressure cell equipped with $\mathrm{CaF}_{2}$ windows by a 6030 Mattson Galaxy Series FT-IR spectrometer, and 64-64 scans were signal-averaged at a resolution of $4 \mathrm{~cm}^{-1}$. Raman spectra for the polymer/silver salt complexes were recorded at room temperature using a Perkin Elmer System 2000 NIR FT-Raman. This experimental apparatus includes a neodymium-doped yttrium aluminum garnet $\left(\mathrm{Nd}^{3+}\right.$ :YAG) laser operating at $1.064 \mu \mathrm{m}$. Spectroscopic characterization was performed using a pressure cell equipped with $\mathrm{CaF}_{2}$ windows.

For the gas permeation test, the polymer solution was coated onto a polyester membrane substrate (Whatman, $0.1 \mu \mathrm{m}$ ) using a RK Control Coater (Model 101, Control Coater RK Print-Coat Instruments LTD, UK). Mixed gas (50:50 vol\% of propylene/propane mixture) separation properties through the membranes were evaluated by gas chromatography (Hewlett Packard G1530A, MA) equipped with a TCD detector. The stage cut $(\theta)$, the ratio of permeate to feed flow rates, was always less than $2 \%$. The unit of gas permeance is GPU, where $1 \mathrm{GPU}=1 \times 10^{-6} \mathrm{~cm}^{3}(\mathrm{STP}) / \mathrm{cm}^{2} \mathrm{~s}$ $\mathrm{cmHg}$ ). The thickness of the top selective layer is ca. $1.5 \mu \mathrm{m}$ as determined by scanning electron microscopy (SEM).

Calculation. The electronic energy (i.e., complexation energy) was calculated by full optimization, without any geometrical constraint, of Beckes three parameter hybrid functional $^{20}$ using the Lee, Yang and Parr correlation functional $^{21}$ with the $6-31+\mathrm{G}(\mathrm{d})$ basis set ${ }^{22}$ for non-metallic elements and the Effective Core Potential (ECP) basis sets of type LANL2DZ for silver cation. The program package Gaussian 98 was used throughout the work.

\section{Results and Discussion}

Electron Density of Polymer Matrix. The charge densities of oxygens in the three polymer matrices were calculated theoretically at B3LYP/6-31G* level and the results are presented in Table I. As seen, the charge densities of two ester oxygens in the three polymers are not significantly different each other, indicating the similar electron donation ability to silver ion and thus expecting the similar interactions of silver salt with three PMAs. Meanwhile, the higher negativity of the charge density for the carbonyl oxygen $(\mathrm{C}=\mathrm{O})$ than $\mathrm{C}-\mathrm{O}$ irrespective of the polymer matrix represents that the silver ions will be more favorably coordinated by the oxygen of $\mathrm{C}=\mathrm{O}$ than that of $\mathrm{C}-\mathrm{O}$ in ester group. It is a good accordance with the previous FTIR spectroscopic results that silver ions are coordinated mainly by the $\mathrm{C}=\mathrm{O}$ oxygen in ester group

Table I. Calculated NBO Charge Density (q) of Ester Oxygen in Electron Unit at B3LYP/6-31G* Level

\begin{tabular}{cccc}
\hline Oxygen Species & PMMA & PEMA & PBMA \\
\hline $\mathrm{C}=\mathrm{O}$ & -0.599 & -0.604 & -0.600 \\
$\mathrm{C}-\mathrm{O}$ & -0.567 & -0.566 & -0.558 \\
\hline
\end{tabular}

for cellulose acetate $(\mathrm{CA}) / \mathrm{AgBF}_{4}$ membranes. ${ }^{10}$

Dissolution of Silver Salts. Despite the similar electron densities of the oxygen in the three polymer matrices, remarkably different physico-chemcial properties for the polymer/silver complex membranes have been achieved. The first is the different dissolution behavior of the silver salt in the polymer matrix depending on the side chain length of alkyl group. WAXS data of the three PMAs and PMA/silver salt complexes were measured and presented in Figure 1. Pure PMA gives rise to a $I(2 \theta)$ vs $2 \theta$ plot with two
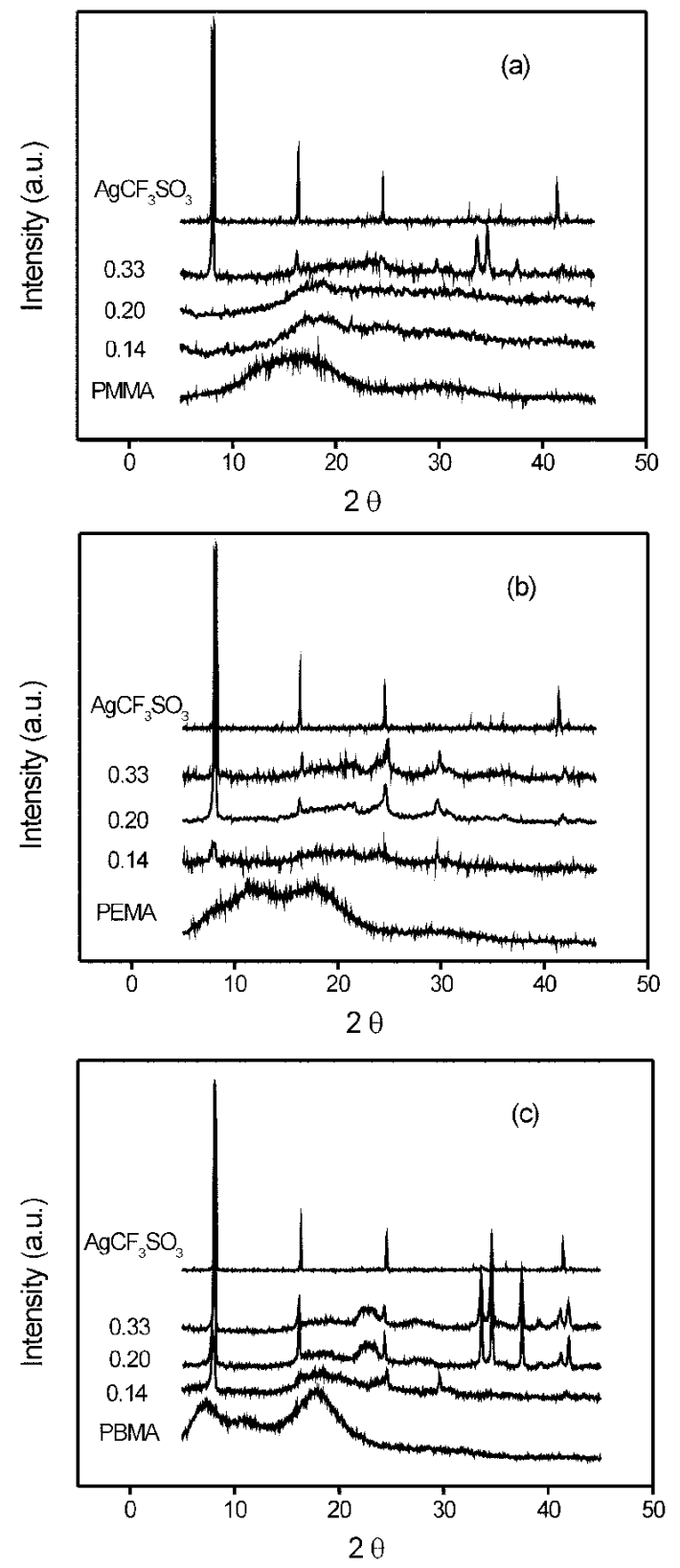

Figure 1. WAXS data of $\mathrm{AgCF}_{3} \mathrm{SO}_{3}$, polymer and polymer/ $\mathrm{AgCF}_{3} \mathrm{SO}_{3}$, complexes with various mole fractions of silver salt. (a) PMMA, (b) PEMA, and (c) PBMA. 
broad amorphous peaks. According to the previous researches, ${ }^{23-26}$ these two peaks could be assigned to the interchain distance and the distance between the pendant groups of PMA. Since PBMA has longer pendant group, two peaks are clearly separated. Furthermore, the Bragg d-spacing of the interchain distance is longer and that of the pendant groups is shorter in PBMA, in compared with those in PEMA and PMMA. Meanwhile, the crystalline peak of $\mathrm{AgCF}_{3} \mathrm{SO}_{3}$ in the PMMA complex is hardly observed up to a silver mole fraction of 0.2 , whereas some crystalline peaks for PEMA and PBMA complexes are shown. This represents that PMMA can dissolve the highest concentration of silver salts among the three PMAs.

The interaction between silver ion and polymeric ligand primarily determines the dissolution ability of silver salt in polymeric ligands and the ionic constituents of silver salts including free ions, ion pairs and higher order aggregates. For instance, free ions are more likely to be formed when the interaction between silver ion and polymeric ligand and consequently the dissolution ability of silver salts in a polymeric ligand are high. Therefore, the interaction is important in the characterization of the ionic constituents. The FTRaman spectra of polymer complexes with $\mathrm{AgCF}_{3} \mathrm{SO}_{3}$ were obtained as a function of silver mole fraction. Figure 2 shows the Raman spectra of the $v_{1}$ symmetric stretching vibration of $\mathrm{SO}_{3}^{-}$for the complexes with (a) PMMA, (b) PEMA and (c) PBMA. According to the previous research, ${ }^{27-29}$ the bands for the $v_{\mathrm{s}}\left(\mathrm{SO}_{3}{ }^{-}\right)$stretching mode at 1032, 1037 and $1048 \mathrm{~cm}^{-1}$ in the complexes are assigned to the free ion, ion pair and higher-order ionic aggregate, respectively. For PMMA/AgCF $\mathrm{SO}_{3}$ complex, free ions are present up to the silver mole fraction of 0.10, as seen in Figure 2(a). When the silver concentration increased further above 0.10 , the main band shifts to a higher wavenumber and the band becomes asymmetric, demonstrating the presence of both ion pairs and free ions. The vibrational FT-Raman spectra of PEMA/ $\mathrm{AgCF}_{3} \mathrm{SO}_{3}$ and $\mathrm{PBMA} / \mathrm{AgCF}_{3} \mathrm{SO}_{3}$ complexes in the $v_{\mathrm{s}}\left(\mathrm{SO}_{3}{ }^{-}\right)$ stretching region show the similar behavior to those of PMMA/AgCF $\mathrm{SO}_{3}$ complex except for the differences in the relative peak intensities of free ions and ion pairs.

To obtain more quantitative information on dissolution ability of polymer matrices for silver salt, the Raman spectra for $\mathrm{PMA} / \mathrm{AgCF}_{3} \mathrm{SO}_{3}$ complexes with various silver mole fractions were deconvoluted shown in Figure 3. The relative concentration of free ions was calculated as the ratio of the deconvoluted area under the peak with maximum value to the total area of the $v_{\mathrm{s}}\left(\mathrm{SO}_{3}^{-}\right)$envelope $\mathrm{e}^{30-32}$ and presented in Figure 4. Irrespective of polymer matrices, only free ions are present up to a silver mole fraction of 0.10 , above which ion pairs start to form and thus the fraction of free ions decreases gradually. The higher concentration of silver salt, the lower concentration of free ions is obtained. More importantly, the concentration of free ions in all silver concentration ranges is in the order of PMMA $>$ PEMA $>$
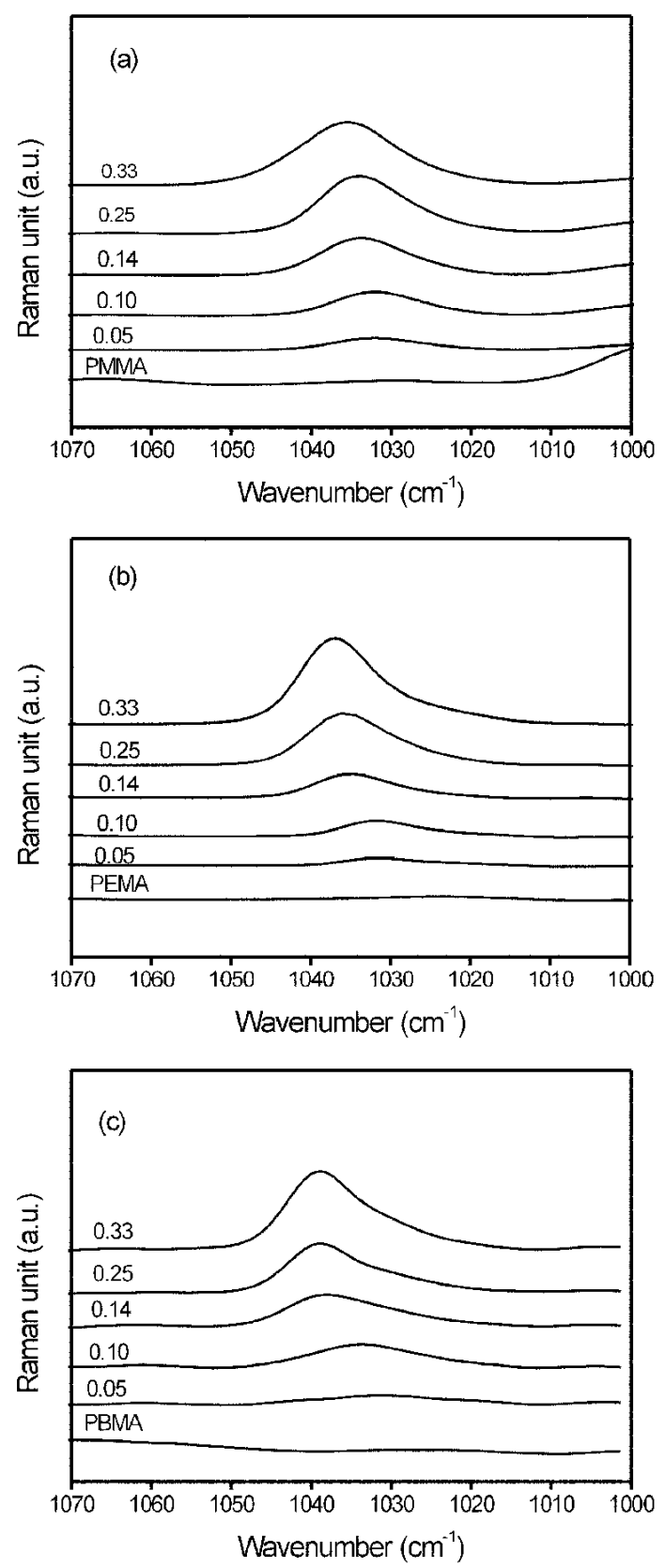

Figure 2. FT-Raman spectra for $\mathrm{SO}_{3}{ }^{-}$stretching region for pure polymer and polymer/ $\mathrm{AgCF}_{3} \mathrm{SO}_{3}$ complexes with various mole fractions of silver salt. (a) PMMA, (b) PEMA, and (c) PBMA.

PBMA. This is because relatively hydrophilic PMMA can dissolve silver salts more than PEMA and PBMA, which is consistent with the WAXS results.

As the alkyl side chain is longer, the hydrophilicity will be decreased: PMMA > PEMA > PBMA, as verified by measuring the contact angles of water for the three polymers (Table II). The lower contact angle of water for PMMA may be associated with the higher concentration of hydrophilic ester groups, representing more hydrophilic. Since silver 

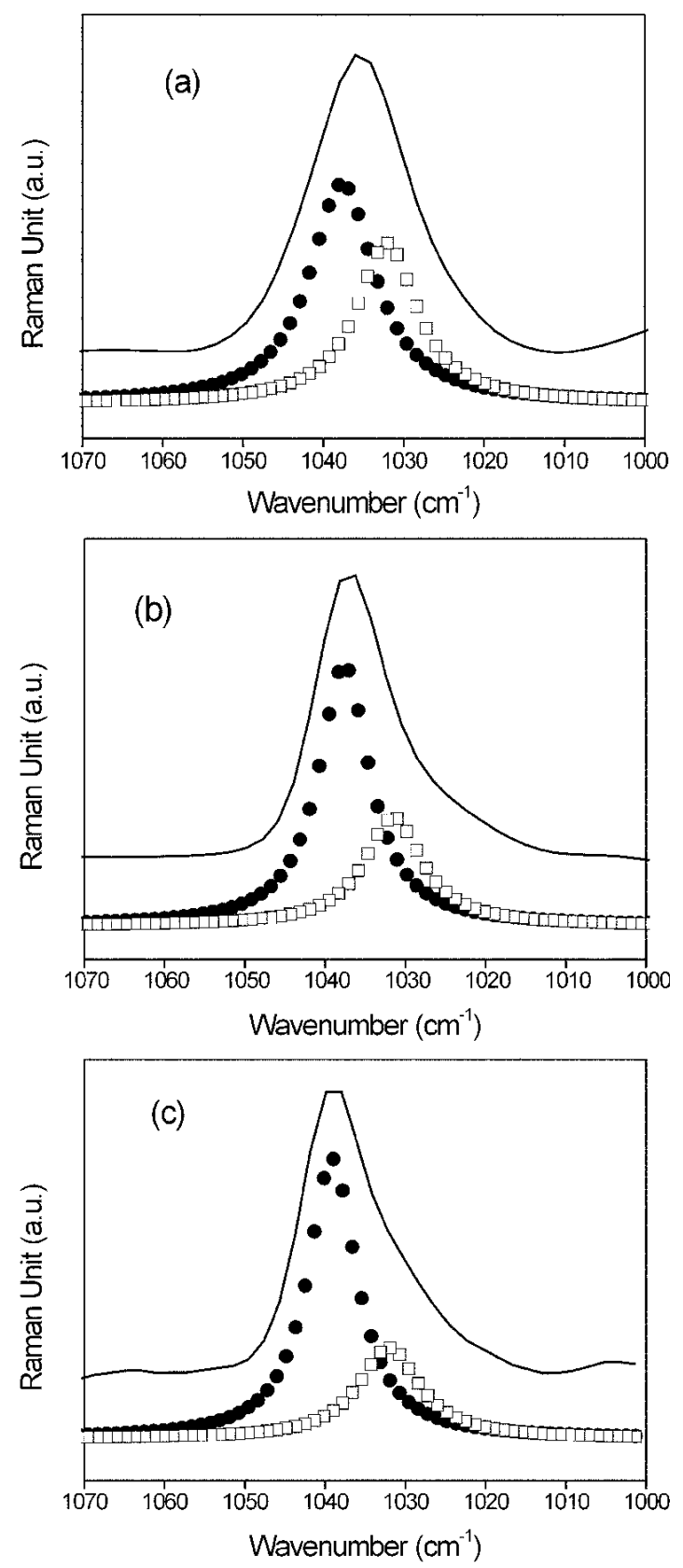

Figure 3. Deconvoluted FT-Raman curves for $\mathrm{SO}_{3}{ }^{-}$stretching region for polymer/ $\mathrm{AgCF}_{3} \mathrm{SO}_{3}$ complexes with a silver mole fraction of 0.33. (a) PMMA, (b) PEMA, and (c) PBMA.

Table II. Contact Angles of Deionized Water for PMMA, PEMA and PBMA

\begin{tabular}{lccc}
\hline & PMMA & PEMA & PBMA \\
\hline Contact Angle & $56^{\circ} \pm 1$ & $72^{\circ} \pm 1$ & $83^{\circ} \pm 1$ \\
\hline
\end{tabular}

salts are also hydrophilic, the hydrophilicity of the polymer matrix might have a positive effect on the dissolution of silver salt. Thus, the relatively hydrophilic PMMA would

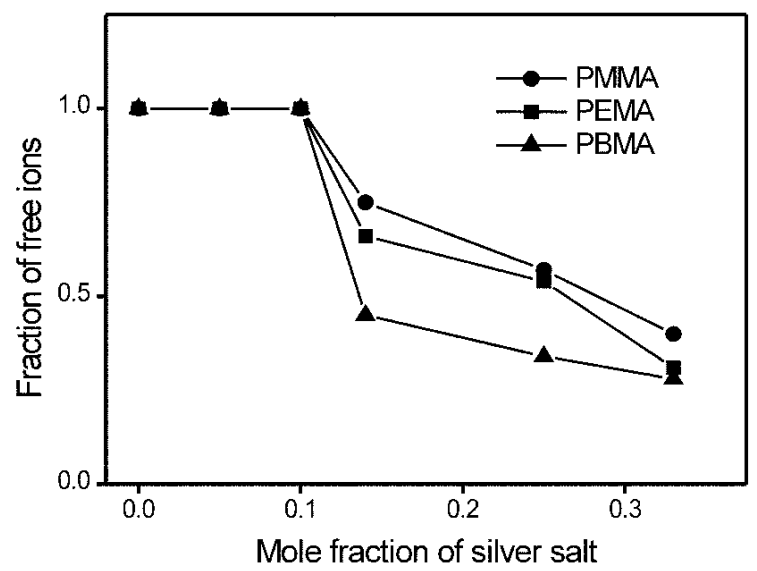

Figure 4. Fraction of free ions in polymer/ $\mathrm{AgCF}_{3} \mathrm{SO}_{3}$ complexes as a function of silver mole fraction.

have strong interaction with silver salts and thus dissolve the higher concentration of the silver salts than PEMA or PBMA.

Facilitated Olefin Transport. It is found that the separation performance through the PMA/silver salt complex membranes is very sensitive to the side chain length. The gas transport properties of propylene/propane mixtures through the three PMA membranes containing $\mathrm{AgBF}_{4}$ and $\mathrm{AgCF}_{3} \mathrm{SO}_{3}$ at $23^{\circ} \mathrm{C}$ were measured and presented as a function of the silver concentration in Figures 5 and 6 . The permeation result clearly indicates that the facilitated olefin transport properties, including the selectivity of propylene/propane and gas permeance, decrease irrespective of the kind of the silver salt: PMMA $>$ PEMA $>$ PBMA. It should be noted that glassy PMMA/silver salt complex membranes show unexpectedly much higher gas permeance than those with rubbery PBMA complex membranes even though neat PMMA is much less permeable than neat PBMA. It is also found that the polymer complex membranes containing $\mathrm{AgBF}_{4}$ showed better separation performance than those containing $\mathrm{AgCF}_{3} \mathrm{SO}_{3}$.

It is well known that the free ion among the three ionic constituents is the most effective olefin carrier and thus the facilitated transport depends primarily on the concentration of the free ion. The propylene permeances depend strongly on the length of the side chain at the same silver more fraction of 0.1 (Figures 5 and 6) even though the free ion concentrations are almost the same (Figure 4). This suggests that the free ion concentration depending on the hydrophilicity of the polymer matrix is important in determining the facilitated olefin transport, but other factors may also play important roles.

Olefin Coordination to Silver Ions. The different olefin solubilities in the three PMA/silver salt complexes were observed by FT-IR spectroscopy. Figure 7 presents the insitu FT-IR spectra of the three PMA membranes containing $\mathrm{AgCF}_{3} \mathrm{SO}_{3}$ with and without propylene sorption. Since the 

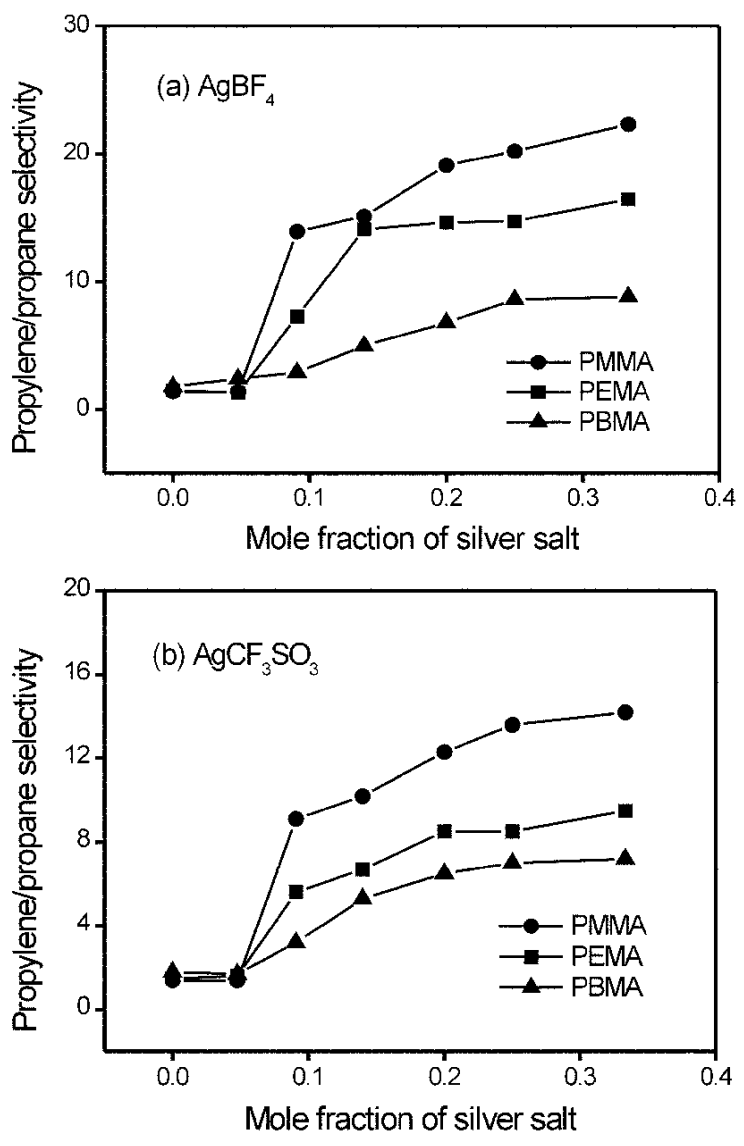

Figure 5. Mixed gas selectivity of propylene/propane through polymer/silver complex membranes as a function of silver mole fraction at $375 \mathrm{kPa}, 23{ }^{\circ} \mathrm{C}$. (a) $\mathrm{AgBF}_{4}$ and (b) $\mathrm{AgCF}_{3} \mathrm{SO}_{3}$.

shape and intensity of the free carbonyl peak in three polymers at $1729 \mathrm{~cm}^{-1}$ nearly coincide each other, only the spectrum of PBMA is plotted for simplicity. For the polymer/ silver salt complex, the spectra of the three membranes before propylene sorption were not significantly different each other, showing two carbonyl peaks of the free $\mathrm{C}=\mathrm{O}$ at $1729 \mathrm{~cm}^{-1}$ and the complexed $\mathrm{C}=\mathrm{O}$ with silver ions at $1700 \mathrm{~cm}^{-1}$. The appearance of the complexed $\mathrm{C}=\mathrm{O}$ peak at a lower wavenumber may result from the loosened $\mathrm{C}=\mathrm{O}$ double bond interaction by the coordinative interaction between silver ions and carbonyl oxygen. However, when the membranes were exposed to $345 \mathrm{kPa}$ of propylene for $30 \mathrm{~min}$ and successively purged with nitrogen for less than one second, the spectra of the three polymer/silver salt complexes were remarkably changed. The intensity of the complexed $\mathrm{C}=\mathrm{O}$ peak with silver ions at $1700 \mathrm{~cm}^{-1}$ was notably decreased. This result strongly suggests that some of the complexed carbonyl oxygens become free, demonstrating that 1) olefins and carbonyl groups compete with each other for the coordination to silver ions, 2) the interaction of silver ion with olefin is equivalent to or slightly stronger than that with ester oxygen. Additionally, a new peak with relatively weak
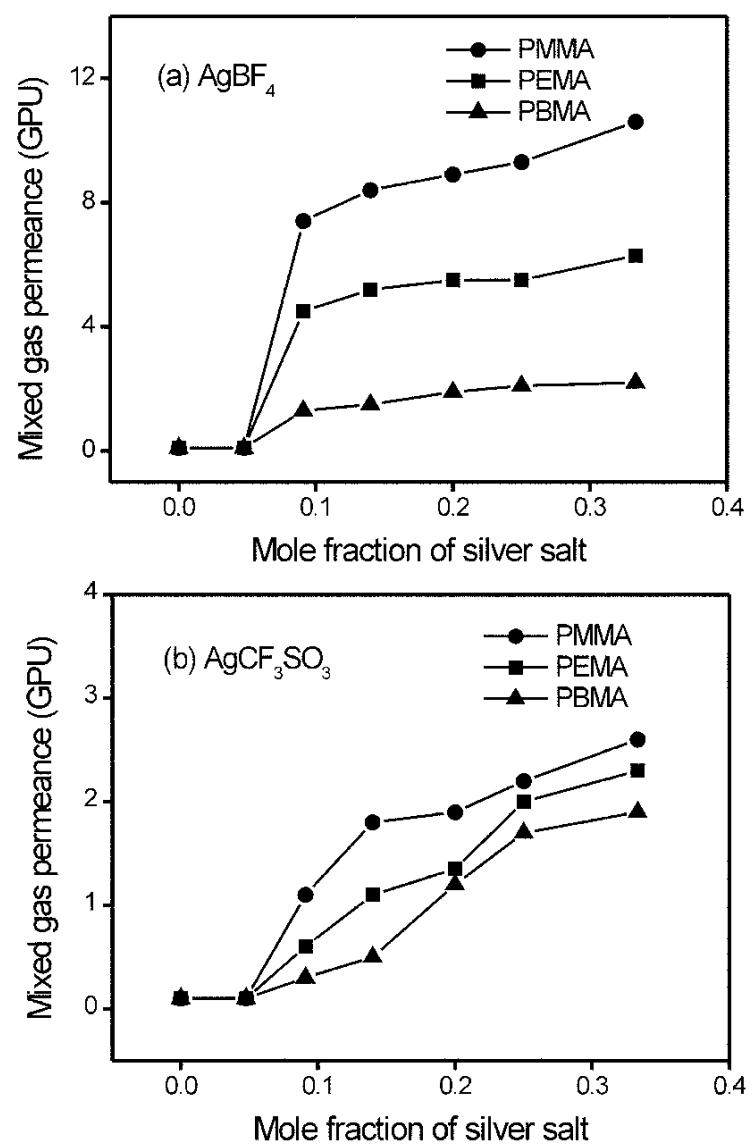

Figure 6. Mixed gas permeance of propylene/propane through polymer/silver complex membranes as a function of silver mole fraction at $375 \mathrm{kPa}, 23^{\circ} \mathrm{C}$. (a) $\mathrm{AgBF}_{4}$ and (b) $\mathrm{AgCF}_{3} \mathrm{SO}_{3}$.

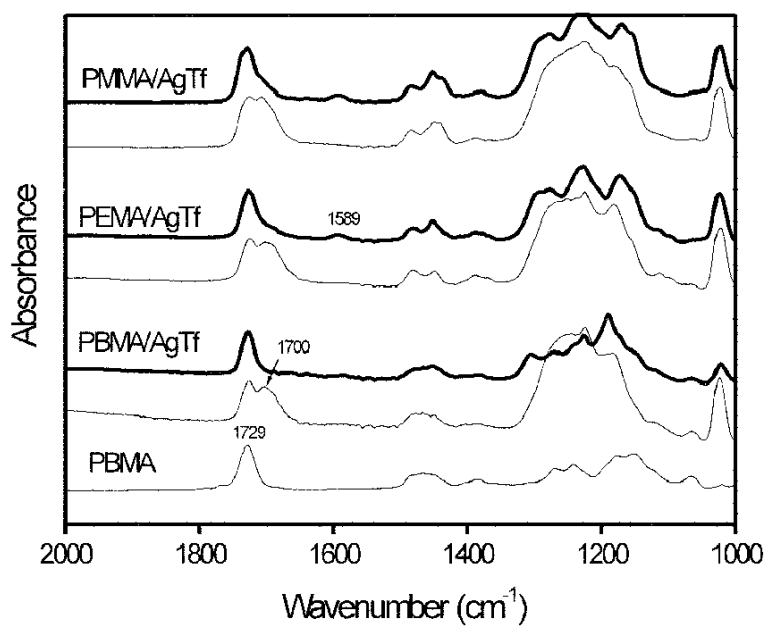

Figure 7. FT-IR spectra for PMMA, PEMA and PBMA complexes containing $\mathrm{AgCF}_{3} \mathrm{SO}_{3}$ with (thick line) and without (thin) propylene sorption at a silver mole fraction of 0.33 . For pure polymer, the spectrum with propylene nearly coincides with that without propylene sorption. 
intensity at $1589 \mathrm{~cm}^{-1}$ appeared, which can be ascribed to the complexed $\mathrm{C}=\mathrm{C}$ of propylene with silver ion. Note that $v_{1}$ and $v_{2}$ of $\mathrm{C}=\mathrm{C}$ in free propylene are 1665 and $1640 \mathrm{~cm}^{-1}$, respectively. The intensity of the new peak could be strongly associated with the amounts of the propylene complexation with silver ions and consequently the propylene solubility in polymer/silver salt complex membranes. ${ }^{7,8}$ Since the intensity of the $\mathrm{C}=\mathrm{C}$ peak at $1589 \mathrm{~cm}^{-1}$ is in the order of PMMA > PEMA $>$ PBMA, it can be obviously demonstrated that the propylene solubility in the polymer/silver complex membranes is arranged as the same order, which is in a good agreement with the trend of facilitated olefin transport.

Role of Side Chain. We have obtained anomalously different propylene solubility and facilitated transport in the PMA/silver complex membranes depending on the side chain length of alkyl group. The three polymer matrices possess the similar molecular structure and oxygen electron density for the possible coordination to silver ions. However, the dissolution ability of polymeric ligand is consistent with the hydrophilicity of PMAs. It is believed that the long side chain connected to the ester group may prohibit the spatial access of olefin molecules to silver ions: steric hindrance. The steric hindrance may also result in the difference in facilitated olefin transport in addition to the hydrophilicity of the polymeric matrices.

\section{Conclusions}

Silver salt complex membranes with glassy PMMA showed unexpectedly much higher propylene permeance than those with rubbery PBMA even though neat PMMA is much less permeable to propylene than neat PBMA. The three PMAs exhibit almost the same charge density of the carbonyl oxygens for the coordination to silver ions. However, the hydrophilicity decreases with the length of the side chain, and may primarily determine the interaction between silver ion and carbonyl oxygen and consequently the free ion concentration. It is also found that both the facilitated olefin transport and olefin solubilities depend strongly on the steric hindrance in addition to the hydrophilicity of the polymeric matrix. Therefore, it is proposed that both the hydrophilicity and the steric hindrance associated with the difference in the side chain length are important in determining facilitated olefin transport through the polymer/silver salt complex membranes.

Acknowledgments. The authors gratefully acknowledge the financial support of the Ministry of Science and Technology of Korea through the Creative Research Initiatives Program.

\section{References}

(1) A. Ferry, G. Orädd, and P. Jacobsson, J. Chem. Phys., 108,
7426 (1998).

(2) S. S. Bae and N. J. Jo, Korea Polym. J., 9, 332 (2001).

(3) C. R. Lee, S. H. Hyun, S. K. Lee, W. S. Kim, S. I. Moon, and B. S. Jin, Korea Polym. J., 9, 292 (2001).

(4) S. U. Hong, J. H. Jin, J. Won, and Y. S. Kang, Adv. Mater., 12, 968 (2000).

(5) J. H. Kim, B. R. Min, C. K. Kim, J. Won, and Y. S. Kang, Macromolecules, 34, 6052 (2001).

(6) J. H. Kim, B. R. Min, C. K. Kim, J. Won, and Y. S. Kang, J. Phys. Chem. B., 106, 2786 (2002).

(7) J. H. Kim, B. R. Min, J. Won, and Y. S. Kang, Chem. Eur. J., 8, 650 (2002).

(8) J. H. Kim, B. R. Min, C. K. Kim, J. Won, and Y. S. Kang, Macromolecules, 35, 5250 (2002).

(9) J. H. Kim, B. R. Min, J. Won, S H. Joo, H. S. Kim, and Y. S. Kang, Macromolecules, 36, 6183 (2003).

(10) J. H. Ryu, H. Lee, Y. J. Kim, Y. S. Kang, and H. S. Kim, Chem. Eur. J., 7, 1525 (2001).

(11) J. H. Kim, B. R. Min, C. K. Kim, J. Won, and Y. S. Kang, J. Polym. Sci., B: Polym. Phys., 40, 1813 (2002).

(12) J. H. Kim, B. R. Min, K. B. Lee, J. Won, and Y. S. Kang, Chem. Commun., 2732 (2002).

(13) J. H. Kim, B. R. Min, H. S. Kim, J. Won, and Y. S. Kang, J. Membr. Sci., 212, 283 (2003).

(14) J. H. Kim, B. R. Min, J. Won, and Y. S. Kang, Macromolecules, 36, 4577 (2003).

(15) I. Pinnau, L. G. Toy, and C. Casillas, U. S. Patent 5,670,051, 1997.

(16) S. Sunderrajan, B. D. Freeman, C. K. Hall, and I. Pinnau, J. Membr. Sci., 182, 1 (2001).

(17) J. Won, Y. Yoon, and Y. S. Kang, Macromol. Res., 10, 80 (2002).

(18) J. M. Hong and Y. S. Kang, Korea Polym. J., 8, 1 (2000).

(19) C. K. Kim, C. K. Kim, B. S. Lee, J. Won, H. S. Kim, and Y. S. Kang, J. Phys. Chem. A., 105, 9024 (2001).

(20) A. D. Becke, J. Chem. Phys., 98, 5648 (1993).

(21) (a) C. Lee, W. Yang, and R. G. Parr, Phys. Rev., B37, 785 (1988); (b) B. Miehlich, A. Savin, H. Stoll, and H. Preuss, Chem. Phys. Lett., 157, 200 (1989).

(22) W. J. Hehre, L. Radom, P. V. R. Schleyer, and J. A. Pople, $A b$ initio Molecular Orbital Theory, John Wiley \& Sons, New York, 1986, Chapter 4.

(23) S. Choi, J. H. Kim, and Y. S. Kang, Marcomolecules, 34, 9087 (2001).

(24) G. R. Mitchell and A. H. Windle, Polymer, 25, 906 (1984).

(25) M. Aguilar-Vega and D. R. Paul, J. Polym. Sci., B: Polym. Phys., 31, 1577 (1993).

(26) R. Lovell and A. Windle, Polymer, 22, 175 (1981).

(27) J. H. Jin, S. U. Hong, J. Won, and Y. S. Kang, Macromolecules, 33, 4932 (2000).

(28) S. Schantz, J. Chem. Phys., 94, 6296 (1991).

(29) J. Manning and R. Frech, Polymer, 33, 3487 (1992).

(30) A. Ferry, P. Jacobsson, and L. M. Torell, Electrochim. Acta, 40, 2369 (1995).

(31) S. Chintapalli and R. Frech, Electrochim. Acta, 43, 1395 (1998).

(32) M. Kakihana, S. Sohantz, and L. M. Torell, J. Chem. Phys., 92, 6271 (1990). 